\title{
The Gender-Specific Effect of Subcutaneous and Visceral Adipose Tissues on Cardiometabolic Risk in a Chinese Population
}

\section{Qiong Wu}

Zhejiang University

\section{Qiannan Chen}

Zhejiang Chinese Medical University

\section{Qingguo LV}

Sichuan University West China Hospital

\section{Sun Xiaohui}

Zhejiang Chinese Medical University

\section{Chen Dai}

Zhejiang Putuo Hospital

\section{Xuhui Zhang}

Hangzhou Center of Disease Control and Prevention

Jiaqiang Zhou

Zhejiang University School of Medicine Sir Run Run Shaw Hospital

Yimin Zhu ( $\nabla$ zhuym@zju.edu.cn)

Zhejiang University https://orcid.org/0000-0001-8409-7636

\section{Research}

Keywords: Obesity, Subcutaneous adipose tissue, Visceral adipose tissue, Cardiometabolic risk factors, Type 2 diabetes Posted Date: January 8th, 2021

DOI: https://doi.org/10.21203/rs.3.rs-139430/v1

License: (9) (1) This work is licensed under a Creative Commons Attribution 4.0 International License. Read Full License 


\section{Abstract}

\section{Background}

Previous studies demonstrated that visceral adipose tissue (VAT) contributed to increased risks for multiple cardiometabolic factors. However, the effects of subcutaneous adipose tissue (SAT) were controversial. Furthermore, their effects were varied across sexes. The aim of this study was to examine the gender-specific effects of VAT, SAT, and VAT/SAT ratio on cardiometabolic risk factors.

\section{Methods}

1388 eligible subjects were recruited from a metabolic syndrome cross-sectional study in China. Areas of abdominal VAT and SAT were determined by nuclear magnetic resonance imaging (MRI). Multiple logistic regression was used to calculate the odds ratios (OR) and $95 \%$ confidence intervals $(95 \% \mathrm{Cl})$.

Results

SAT, VAT, and VAT/SAT ratio had significant age-adjusted, positive correlations with body mass index (BMI), waist circumference (WC), waist-to-hip ratio (WHR), systolic blood pressure (SBP), diastolic blood pressure (DBP), fasting plasma glucose (FPG), total triglycerides (TG), and inverse correlations with high-density lipoprotein cholesterol (HDL-C) except VAT/SAT ratio with FPG in men. After additional controlled by BMI, consistent results were found in VAT and VAT/SAT ratio except for VAT with FPG, however, women showed significant inverse correlations between SAT and SBP, DBP and TG. Higher SAT exhibited a significantly reduced risk for type 2 diabetes (T2D) in women, the ORs in third quartile (Q3) and fourth quartile (Q4) group were 0.3 (95\% Cl: 0.13-0.74) and 0.19 (95\% Cl: 0.07-0.54). Men had higher ORs of VAT, VAT/SAT ratio than women in higher TG, low HDL-C, and hypertension while the ORs of VAT/SAT ratio in men were lower than women in T2D.

\section{Conclusions}

SAT exhibited a beneficial effect on T2D. VAT and VAT/SAT ratio linked increased risks for high TG, low HDL-C, and hypertension, with men had a more pronounced effect than women. Distribution of abdominal fat associates with its biological effects.

\section{Introduction}

Obesity, especially central obesity, was a well-established risk factor of insulin resistance, dyslipidemia, type 2 diabetes, cardiovascular diseases, and all-cause mortality[1, 2]. BMI and WC, as useful indicators of body mass, have been widely used to evaluate obesity, however, due to the difference in body shapes, both the two indices cannot capture the true effect of regional fat depots[3]. The accurate assessment of regional fat depots currently still requires imaging with radiographic techniques such as computed tomography or magnetic resonance imaging[3].

Increasing evidence has suggested that VAT was an endocrine organ and had deleterious impacts on multiple cardiometabolic risk factors by secreting amounts of adipocytokines and vasoactive substances[4,5]. While reports on the effects of SAT on metabolic abnormality still inconclusive or even contradictive [1, 6-8]. Some studies demonstrated that SAT was a beneficial fat depot for type 2 diabetes, metabolic syndrome[8, 9], However, other studies did not find any significant associations between SAT and metabolic abnormality $[6,7,10]$. Moreover, given that the fat depot accumulation was different between sexes, their effects on the metabolic abnormality also varied. Some studies found that higher VAT was causally associated with cardiometabolic factors with a larger effect in women[11]. While other studies indicated that VAT contributes to a more absolute risk for metabolic factors in men than women[12], highlighting the gender difference in the contribution of regional fat distribution. 
A possible reason for these sophisticated associations may due to the distinct fat distribution across ethnic groups[13, 14]. In addition, the way of measurement of SAT and VAT, and adjusted confounding factors were also likely reasons. In the Chinese population, limited studies had probed into the effect of MRI-measured SAT, VAT on metabolic disorders in different sex.

Thus, the aim of this study was to investigate the gender-specific associations of SAT, VAT measured by MRI, with metabolic abnormalities in a Chinese population. In addition, VAT/SAT ratio, which represented the relative fat distribution, will be also examined in this study.

\section{Methods}

\section{Population}

The subjects were recruited from our previous cross-sectional investigation on metabolic syndrome in 2010. The detailed information was previously described [15]. In this study, a subpopulation from two communities in Hangzhou $(n=1170)$ and Chengdu ( $n=761)$ was included. Subjects were excluded if they had 1) severe chronic diseases including cardiovascular diseases, cancers, kidney dysfunction and other wasting diseases, 2) missing anthropometric data, 3) missing data of areas of visceral or subcutaneous fat adipose tissue. Consequently, a total of 1388 eligible subjects were included.

The project was approved by the institutional review board at Zhejiang University, Zhejiang, China. All participants were given their written informed consent.

\section{SAT and VAT measurements}

Abdominal adipose tissue was measured by nuclear magnetic resonance imaging (MRI) using a whole-body imaging system (SMT-100, Shimadzu Co, Japan) with TR-500 and TE-200 of SE. MRI scan was performed at the level of umbilicus between L4 and L5 with the subject in the supine position. The areas of subcutaneous abdominal adipose tissue and visceral abdominal adipose tissue were calculated using SliceOmatic software (version 4.2).

\section{Covariant assessment}

With a standard questionnaire, the information including age, gender, smoking (current, former, and never), alcohol drinking behaviors (never, moderate, and heavy), and menstrual history (for women) were collected. Current smoking was defined as smoking at least one cigarette per day and lasting for one year. Former smoking was defined as quitting smoking for at least one year. Heavy drinking was defined as drinking more than three times per week. Moderate drinking was defined as drinking between the levels of heavy and never. Anthropometric variables were collected by trained investigators with a standard protocol, including weight, height, WC, systolic blood pressure, and diastolic blood pressure. Body mass index was defined as weight (in kilograms) divided by the square of height (in meters). WC was measured at the midpoint between the iliac crest and lowest rib. Blood pressure was measured in a sitting position with a mercury sphygmomanometer. SBP and DBP were measured as the average of three repeat measurements with at least 30-s intervals.

The overnight fasting blood samples were collected for each subject. Total triglycerides, total cholesterol (TC), high-density lipoprotein cholesterol, low-density lipoprotein cholesterol (LDL-C) were measured by biochemical auto-analyzers (Hitachi 7060, Tokyo, Japan). Fasting plasma glucose was analyzed with a glucose oxidase method with the Beckman Glucose Analyzer (Beckman Instruments, Irvine, CA, USA).

High TG was defined as $>1.7 \mathrm{~mm} / \mathrm{L}$, low HDL-C was defined as $<1.03 \mathrm{~mm} / \mathrm{L}$ (in men), $<1.29 \mathrm{~mm} / \mathrm{L}$ (in women); Type 2 diabetes was defined as fasting blood glucose $>7.0 \mathrm{mmol} / \mathrm{L}$ or 2 hour post oral glucose tolerance test (OGTT $2 \mathrm{~h}$ ) $>11.0 \mathrm{mmol} / \mathrm{L}$, or have a history of diabetes, or using drugs of anti-diabetes. Hypertension was defined as SBP $>140 \mathrm{mmHg}$, or $\mathrm{DBP}<90 \mathrm{mmHg}$ or using antihypertensive drug therapy.

\section{Statistical analysis}


Continuous variables were presented as mean \pm standard deviation or medians (inter-quartiles range). Categorical variables were shown as number (\%). Student's t-test or the Wilcoxon rank-sum test was used to compare continuous variables. Chisquare test was used to compare categorical variables. Spearman partial correlations between SAT, VAT, VAT/SAT ratio and cardiometabolic factors were analyzed, adjusted for age or age and BMI. The subjects were divided into four groups by quartiles of SAT, VAT, and VAT/SAT ratio, with the first quartile (Q1) as the reference group. The ORs and $95 \% \mathrm{Cls}$ for each quartile by sex were calculated using multiple logistic regression, adjusted for age, smoking, alcohol consumption, menstrual history (for women) (model1) and variables in model1 plus BMI (model2).

The significance level was set at $P<0.05$. The software IBM SPSS Statistics version 25.0 was used to analyze the data.

\section{Results}

\section{The baseline characteristics of subjects}

The baseline characteristics by sex are summarized in Table1. Of the 1388 subjects, $622(44.81 \%)$ were men, 766 (55.19\%) were women. The mean age was 53.8 years old. The mean BMI, SAT, VAT, VAT/SAT ratio were 23.70 $\pm 2.99,148.45(112.90-$ 194.70), 69.51(45.54-107.20), and 0.45(0.30-0.69), respectively. Compared with women, men were more likely to have higher BMI, WC, hip circumference (HC), WHR, VAT, VAT/SAT ratio, SBP, DBP, FPG, OGTT-2h, TG, and a higher prevalence of high TG, hypertension, and T2D. Women had higher SAT, TC, and HDL-C.

\section{The correlations between SAT, VAT, VAT/SAT and cardiometabolic factors}

Table 2 presents the correlations between SAT, VAT, VAT/SAT ratio and cardiometabolic factors adjusted by age or age and BMI stratified by sex. SAT, VAT, and VAT/SAT ratio had significant age-adjusted and positive correlations with BMI, WC, WHR, SBP, DBP, FPG, TG, and inverse correlations with HDL-C (all the P values < 0.05) except VAT/SAT ratio with FPG in men $(P>$ 0.05). After additional controlled by BMI, consistent results were found in VAT and VAT/SAT ratio except for VAT with FPG, however, women showed significant inverse correlations between SAT and SBP, DBP, and TG. No significance in HDL-C and FPG was found in women. In men, also, no significant correlation was found in SBP and FPG.

\section{The associations between SAT, VAT, VAT/SAT ratio and the risks of cardiometabolic abnormalities}

Figure 1A and Figure 1B presents that in both men and women, higher levels of SAT, VAT, and VAT/SAT ratio were significantly associated with higher risks for high TG, low HDL-C, hypertension ( $p$ for trend $<0.05$ ) except no significant association between SAT and HDL-C in women. Men showed a significant dose-response association between SAT and the risk for T2D ( $p$ for trend $=0.035$ ), but no significance was found in VAT and VAT/SAT ratio for T2D. Higher levels of VAT and VAT/SAT ratio in women displayed an elevated risk for T2D, but no significant risk was found in SAT.

After additionally adjusted for BMI, the dose-dependent association between VAT and T2D in women disappeared, the other associations of VAT and VAT/SAT ratio with metabolic abnormalities remained significant ( $\mathrm{f}$ for trend $<0.05$, Figure $2 \mathrm{~A}$ and Figure 2B). No significant dose-response relationship of SAT with high TG, low HDL, and hypertension were found in both men and women. Higher SAT seemed significantly reduced risk for T2D in women ( $p$ for trend $<0.01$ ). The subjects had the OR of 0.3 (95\% Cl: 0.13-0.74) for T2D in the Q3 group and 0.19 (95\% Cl: 0.07-0.54) in the Q4 group.

Table3 presents the associations of Z score-transformed SAT and VAT with the risks for metabolic abnormalities in men and women, which shows that men had higher ORs of VAT, VAT/SAT ratio than women in higher TG, low HDL-C, and hypertension while the effects of VAT/SAT ratio in men were lower than women in T2D. Significant interactions were found between SAT or VAT/SAT, and T2D $(P<0.05)$, a borderline significance of VAT in higher TG was observed $(P<0.1)$. Consistent results were found in the analyses with or without additional adjustment for BMI.

\section{Discussion}


In this cross-sectional study, we found higher VAT and VAT/SAT ratio rather than SAT linked to increased risk for high TG, low HDL-C, and hypertension beyond BMI, with men had higher effects on these metabolic abnormalities. For the risk of T2D, higher SAT exerted a protective effect in women independent of BMI. VAT did not have any relationship with T2D in both genders. Higher VAT/SAT ratio showed a significant positive association with T2D in women, independent of BMI. Significant interactions were found between SAT or VAT/SAT, and T2D. Borderline sex interaction of VAT in higher TG was observed.

It is well established that VAT exerted an adverse effect on several metabolic risk factors, and may have a unique pathogenic property beyond BMI $[1,7,16,17]$. Our study confirmed this finding. VAT was a metabolically active organ, which included more non-adipocytes including macrophages, immune cells, preadipocytes and fibroblasts, and could secrete amounts of inflammation mediators to induce metabolism impairments $[4,5,18,19]$. In addition, high lipolytic activity in VAT and its accompanied inflammation response also contributed to abnormal lipogenesis, glucose homeostasis, and vascular health[20, 21]. Therefore, it is plausible that higher VAT volume increased the risk for these metabolic diseases. However, in this study, no significant association between VAT and T2DM risk was found, which may be a consequence of confounding, such as the history of medication, physical activity, dietary, and so on.

With regards to the contribution of VAT in different sex, inconclusive results were reported[11, 12, 17, 22, 23]. Several Caucasian population studies showed the associations of VAT with type 2 diabetes, hypertension and hyperlipidemia were stronger in women[11, 17,24]. While other studies reported similar correlations in both genders or stronger associations in men between VAT and some risk factors [12, 22] [25]. In this study, we observed that the effect of VAT on high TG was higher in men, indicating that VAT may have more striking effect on the lipid metabolism in men than women. For T2D, consistent with previous studies, women showed a more adverse effect of VAT than men, with per SD increase of VAT, the ORs (95\%Cls) in women and men were $1.35(0.98,1.86)$ and 1.02(0.75, 1.39), respectively, but no significant sex interaction was found, which may attribute to our low sample size. Another possible reason was that only limited confounding factors were adjusted, which may bias the results. Extending studies in Chinese population needed to determine the gender difference in the contribution of VAT.

Conflicting results were generated for the effect of SAT on cardiometabolic factors[1, 7, 14, 17, 26, 27]. In line with previous studies[26, 28, 29], our study showed higher SAT did not link to increased risk for hypertension, higher TG, and lower HDL-C beyond BMI. However, several studies from European or African population found that SAT had independent associations with high blood pressure and HDL-C[1, 3, 22], underscoring the distinct effect of SAT across ethnics. For its contribution to T2D, some researchers reported that higher SAT levels were positively associated with 2-h plasma glucose, insulin levels in men, while other studies found that higher SAT was associated with reduced risk for metabolic syndrome and T2D in women[6, 26, 30], and no relevance was found in men, suggesting the gender difference of the effect of SAT. Also, there were some studies did not observe a significant association of SAT with the risk of prediabetes and T2D[7, 17, 27], but some of them did not control the effect of BMI[27]. Therefore, the existing ambiguous results may because of the study population and/or the adjusted confounding factors.

A possible explanation for this gender difference in SAT was the distinct sex steroid hormone profiles since these sex hormones are important factors in regulating the adipose tissue distribution and energy metabolism[31, 32]. For the protective effect of SAT, there were also several hypotheses to explain this observation. One is with smaller adipocyte, SAT was more insulin sensitive, and have a larger capacity to uptake fatty acids and triglycerides, thus can act as a powerful buffer to prevent excess fat into non-adipose tissue[30]. On the other hand, SAT could secret more favorable adipokines such as adiponectin, which had anti-diabetes and anti-atherogenic properties[4,20]. Animal studies also showed that the transplantation of SAT into abdominal tissue improved insulin sensitivity [33]. Thus, SAT may be an important metabolic sink for diabetes in women.

Our study has some advantages. The precise and reliable measurement of SAT and VAT is a markable advantage of this study, another is that the assessment of T2D was based on the OGTT-2h glucose and fasting glucose. Our study also has some limitations. First, we cannot infer causality between the fat indices and the metabolic risk factors because of the cross- 
sectional design. Second, limited confounding factors were included in this study, for example, regional fat distribution, such as deep SAT and superficial SAT, and drug use were not included, which may bias the results. Thirdly, the sample size of this study was relatively small, especially for the analysis of T2D. Finally, our data were based on only one single ethnic group, thus the results may not be applied to other ethnicities.

\section{Conclusion}

SAT exhibited a beneficial effect on T2D. VAT and VAT/SAT ratio linked increased risks for high TG, low HDL-C, and hypertension, with men had a more pronounced effect than women. Distribution of abdominal fat associates with its biological effect

\section{Abbreviations}

SAT, subcutaneous adipose tissue; VAT, visceral adipose tissue; MRI, nuclear magnetic resonance imaging; BMI, body mass index; WC, waist circumference; WHR, waist-to-hip ratio; SBP, systolic blood pressure; DBP, diastolic blood pressure; FPG, fasting plasma glucose; OGTT-2h, 2 hour post oral glucose tolerance test; TC, total cholesterol; TG, triglyceride; HDL-C, high density lipoprotein cholesterol, LDL-C, low density lipoprotein cholesterol; T2D, type 2 Diabetes.

\section{Declarations}

\section{Acknowledgement}

We also would like to thank all the participants and investigators that took part in this study.

\section{Funding Statement}

This work was supported by the grants from National Key Research and Development Program of China (2017YFC0907004), Hangzhou Science and Technology Project (20171226Y27), and Zhejiang Health Science and technology Project (2021KY268). The funder has no role in the design of the study, collection, analysis, and interpretation of data.

\section{Availability of data and materials}

The datasets used and/or analyzed during the current study are available from the corresponding author on reasonable request.

\section{Conflict of Interest}

The authors declare that they have no competing interests.

\section{Ethics approval and consent to participate}

The project was approved by the institutional review board at Zhejiang University, Zhejiang, China. All participants were given their written informed consent.

\section{Authors' contributions}

Conceptualization, Yimin Zhu; Data curation, Qiannan Chen; Formal analysis, Qiong Wu and Qiannan Chen; Funding acquisition, Xuhui Zhang and Yimin Zhu; Investigation, Qingguo Lv, Xiaohui Sun, Chen Dai, and Jiaqiang Zhou; Project administration, Xuhui Zhang and Yimin Zhu; Supervision, Yimin Zhu; Writing - original draft, Qiong Wu and Qiannan Chen; Writing - review \& editing, Xuhui Zhang and Yimin Zhu.

\section{References}


[1] Abraham TM, Pedley A, Massaro JM, Hoffmann U, Fox CS. Association between visceral and subcutaneous adipose depots and incident cardiovascular disease risk factors. Circulation 2015;132(17):1639-47.

[2] Rothney MP, Catapano AL, Xia J, Wacker WK, Tidone C, Grigore L, et al. Abdominal visceral fat measurement using dualenergy X-ray: association with cardiometabolic risk factors. Obesity (Silver Spring, Md) 2013;21(9):1798-802.

[3] Bertoli S, Leone A, Vignati L, Spadafranca A, Bedogni G, Vanzulli A, et al. Metabolic correlates of subcutaneous and visceral abdominal fat measured by ultrasonography: a comparison with waist circumference. Nutr J 2016;15:2.

[4] Ibrahim MM. Subcutaneous and visceral adipose tissue: structural and functional differences. Obes Rev 2010;11(1):11-8.

[5] Despres JP, Lemieux I, Bergeron J, Pibarot P, Mathieu P, Larose E, et al. Abdominal obesity and the metabolic syndrome: contribution to global cardiometabolic risk. Arterioscler Thromb Vasc Biol 2008;28(6):1039-49.

[6] Chen P, Hou X, Hu G, Wei L, Jiao L, Wang H, et al. Abdominal subcutaneous adipose tissue: a favorable adipose depot for diabetes? Cardiovascular diabetology 2018;17(1):93.

[7] Liu J, Fox CS, Hickson DA, May WD, Hairston KG, Carr JJ, et al. Impact of abdominal visceral and subcutaneous adipose tissue on cardiometabolic risk factors: the Jackson Heart Study. J Clin Endocrinol Metab 2010;95(12):5419-26.

[8] Kwon H, Kim D, Kim JS. Body Fat Distribution and the Risk of Incident Metabolic Syndrome: A Longitudinal Cohort Study. Sci Rep 2017;7(1):10955.

[9] Porter SA, Massaro JM, Hoffmann U, Vasan RS, O'Donnel CJ, Fox CS. Abdominal subcutaneous adipose tissue: a protective fat depot? Diabetes care 2009;32(6):1068-75.

[10] Hoyer D, Boyko EJ, McNeely MJ, Leonetti DL, Kahn SE, Fujimoto WY. Subcutaneous thigh fat area is unrelated to risk of type 2 diabetes in a prospective study of Japanese Americans. Diabetologia 2011;54(11):2795-800.

[11] Karlsson T, Rask-Andersen M, Pan G, Höglund J, Wadelius C, Ek WE, et al. Contribution of genetics to visceral adiposity and its relation to cardiovascular and metabolic disease. Nat Med 2019;25(9):1390-5.

[12] Chen Y, Zhang Z, Wang J, Sun H, Zhao X, Cheng X, et al. Sex differences in the association of abdominal adipose tissue and anthropometric data with untreated hypertension in a Chinese population. Biology of sex differences 2020;11(1):38.

[13] Lesser IA, Gasevic D, Lear SA. The effect of body fat distribution on ethnic differences in cardiometabolic risk factors of Chinese and Europeans. Appl Physiol Nutr Metab 2013;38(7):701-6.

[14] Rønn PF, Andersen GS, Lauritzen T, Christensen DL, Aadahl M, Carstensen B, et al. Ethnic differences in anthropometric measures and abdominal fat distribution: a cross-sectional pooled study in Inuit, Africans and Europeans. Journal of epidemiology and community health 2017;71(6):536-43.

[15] Zheng R, Yang M, Bao Y, Li H, Shan Z, Zhang B, et al. Prevalence and Determinants of Metabolic Health in Subjects with Obesity in Chinese Population. International journal of environmental research and public health 2015;12(11):13662-77.

[16] Oka R, Miura K, Sakurai M, Nakamura K, Yagi K, Miyamoto S, et al. Impacts of visceral adipose tissue and subcutaneous adipose tissue on metabolic risk factors in middle-aged Japanese. Obesity (Silver Spring, Md) 2010;18(1):153-60.

[17] Fox CS, Massaro JM, Hoffmann U, Pou KM, Maurovich-Horvat P, Liu CY, et al. Abdominal visceral and subcutaneous adipose tissue compartments: association with metabolic risk factors in the Framingham Heart Study. Circulation 2007;116(1):39-48. 
[18] Kahn CR, Wang G, Lee KY. Altered adipose tissue and adipocyte function in the pathogenesis of metabolic syndrome. The Journal of clinical investigation 2019;129(10):3990-4000.

[19] Misra A, Vikram NK. Clinical and pathophysiological consequences of abdominal adiposity and abdominal adipose tissue depots. Nutrition (Burbank, Los Angeles County, Calif) 2003;19(5):457-66.

[20] Chait A, den Hartigh LJ. Adipose Tissue Distribution, Inflammation and Its Metabolic Consequences, Including Diabetes and Cardiovascular Disease. Frontiers in cardiovascular medicine 2020;7:22.

[21] Després JP, Lemieux I. Abdominal obesity and metabolic syndrome. Nature 2006;444(7121):881-7.

[22] Rønn PF, Andersen GS, Lauritzen T, Christensen DL, Aadahl M, Carstensen B, et al. Abdominal visceral and subcutaneous adipose tissue and associations with cardiometabolic risk in Inuit, Africans and Europeans: a cross-sectional study. BMJ open 2020;10(9):e038071.

[23] Bidulescu A, Liu J, Hickson DA, Hairston KG, Fox ER, Arnett DK, et al. Gender differences in the association of visceral and subcutaneous adiposity with adiponectin in African Americans: the Jackson Heart Study. BMC Cardiovasc Disord 2013;13:9.

[24] Borel AL, Nazare JA, Smith J, Aschner P, Barter P, Van Gaal L, et al. Visceral, subcutaneous abdominal adiposity and liver fat content distribution in normal glucose tolerance, impaired fasting glucose and/or impaired glucose tolerance. Int $\mathrm{J}$ Obes (Lond) 2015;39(3):495-501.

[25] Scheuer SH, Faerch K, Philipsen A, Jorgensen ME, Johansen NB, Carstensen B, et al. Abdominal Fat Distribution and Cardiovascular Risk in Men and Women With Different Levels of Glucose Tolerance. J Clin Endocrinol Metab 2015;100(9):3340-7.

[26] Matsha TE, Ismail S, Speelman A, Hon GM, Davids S, Erasmus RT, et al. Visceral and subcutaneous adipose tissue association with metabolic syndrome and its components in a South African population. Clin Nutr ESPEN 2019;32:76-81.

[27] Tang L, Zhang F, Tong N. The association of visceral adipose tissue and subcutaneous adipose tissue with metabolic risk factors in a large population of Chinese adults. Clin Endocrinol (Oxf) 2016;85(1):46-53.

[28] Koh H, Hayashi T, Sato KK, Harita N, Maeda I, Nishizawa Y, et al. Visceral adiposity, not abdominal subcutaneous fat area, is associated with high blood pressure in Japanese men: the Ohtori study. Hypertens Res 2011;34(5):565-72.

[29] Zhao X, Gang X, Liu Y, Sun C, Han Q, Wang G. Using Metabolomic Profiles as Biomarkers for Insulin Resistance in Childhood Obesity: A Systematic Review. Journal of diabetes research 2016;2016:8160545.

[30] Kim S, Cho B, Lee H, Choi K, Hwang SS, Kim D, et al. Distribution of abdominal visceral and subcutaneous adipose tissue and metabolic syndrome in a Korean population. Diabetes care 2011;34(2):504-6.

[31] Palmer BF, Clegg DJ. The sexual dimorphism of obesity. Molecular and cellular endocrinology 2015;402:113-9.

[32] Karastergiou K. The Interplay Between Sex, Ethnicity, and Adipose Tissue Characteristics. Curr Obes Rep 2015;4(2):26978.

[33] Tran TT, Yamamoto Y, Gesta S, Kahn CR. Beneficial effects of subcutaneous fat transplantation on metabolism. Cell Metab 2008;7(5):410-20.

\section{Tables}

Table 1. The baseline characteristics of the participants in this study 


\begin{tabular}{|c|c|c|c|c|}
\hline Characteristics & Overall(n=1388) & Men $(n=622)$ & Women $(n=766)$ & $\mathrm{P}$ \\
\hline Age $\Downarrow$ years $\rrbracket$ & $53.8 \pm 7.1$ & $53.6 \pm 7.1$ & $53.9 \pm 7.1$ & 0.539 \\
\hline BMI $\left(\mathrm{kg} / \mathrm{m}^{2}\right)$ & $23.70 \pm 2.99$ & $24.18 \pm 2.99$ & $23.31 \pm 2.93$ & $<0.001$ \\
\hline WC (cm) & $79.27 \pm 9.00$ & $83.28 \pm 8.39$ & $76.00 \pm 8.12$ & $<0.001$ \\
\hline WHR & $0.87 \pm 0.07$ & $0.90 \pm 0.06$ & $0.84 \pm 0.06$ & $<0.001$ \\
\hline SAT area $\left(\mathrm{cm}^{2}\right)$ & $148.45(112.90-194.70)$ & $123.15(98.05-149.76)$ & $178.20(139.08-221.63)$ & $<0.001$ \\
\hline VAT area $\left(\mathrm{cm}^{2}\right)$ & 69.51(45.54-107.20) & $90.99(55.12-127.43)$ & 60.36(43.30-79.62) & $<0.001$ \\
\hline VAT / SAT ratio & $0.45(0.30-0.69)$ & $0.69(0.48-0.93)$ & $0.33(0.25-0.46)$ & $<0.001$ \\
\hline $\mathrm{SBP} \rrbracket \mathrm{mmHg} \rrbracket$ & $120.46 \pm 15.81$ & $123.72 \pm 15.44$ & $117.82 \pm 15.62$ & $<0.001$ \\
\hline $\mathrm{DBP} \rrbracket \mathrm{mmHg} \rrbracket$ & $79.53 \pm 9.82$ & $82.44 \pm 9.76$ & $77.17 \pm 9.22$ & $<0.001$ \\
\hline FPG (mmol/L) & $5.12 \pm 1.17$ & $5.23 \pm 1.41$ & $5.03 \pm 0.92$ & 0.003 \\
\hline OGTT-2h (mmol/L) & $6.65 \pm 3.30$ & $6.97 \pm 3.93$ & $6.38 \pm 2.65$ & 0.002 \\
\hline $\mathrm{TC}(\mathrm{mmol} / \mathrm{L})$ & $5.28 \pm 1.08$ & $5.13 \pm 1.00$ & $5.40 \pm 1.13$ & $<0.001$ \\
\hline $\mathrm{TG}(\mathrm{mmol} / \mathrm{L})$ & $0.13 \pm 0.25$ & $0.19 \pm 0.27$ & $0.08 \pm 0.23$ & $<0.001$ \\
\hline $\mathrm{HDL}-\mathrm{C}(\mathrm{mmol} / \mathrm{L})$ & $1.48 \pm 0.36$ & $1.36 \pm 0.33$ & $1.58 \pm 0.36$ & $<0.001$ \\
\hline LDL-C(mmol/L) & $2.59 \pm 0.67$ & $2.57 \pm 0.67$ & $2.61 \pm 0.66$ & 0.273 \\
\hline High TG $(n, \%)$ & $459(33.1)$ & $262(42.1)$ & 197(25.7) & $<0.001$ \\
\hline Low HDL-C (n, \%) & 265(19.1\%) & 111(17.8) & 154(20.1) & 0.287 \\
\hline Hypertension (n, \%) & $413(29.8)$ & $225(36.2)$ & $188(24.5)$ & $<0.001$ \\
\hline T2D (n, \%) & $144(9.2 \%)$ & $88(14.1)$ & $56(7.3)$ & $<0.001$ \\
\hline
\end{tabular}

Data are presented as means \pm standard deviation or medians (inter-quartile ranges) or $\mathrm{n}$ (percentage). BMI, body mass index, WC, waist circumference; WHR, waist-to-hip ratio; SAT, subcutaneous adipose tissue; VAT, visceral adipose tissue; SBP, systolic blood pressure; DBP, diastolic blood pressure; FPG, fasting plasma glucose; OGTT-2h, 2 hour post oral glucose tolerance test; TC, total cholesterol; TG, triglyceride; HDL-C, high density lipoprotein cholesterol, LDL-C, low density lipoprotein cholesterol; T2D, type 2 Diabetes.

Table 2. The sex-specific spearman correlation coefficients of SAT, VAT, VAT/SAT ratio with the metabolic risk factors 


\begin{tabular}{|c|c|c|c|c|c|c|}
\hline & \multicolumn{2}{|l|}{ SAT } & \multicolumn{2}{|l|}{ VAT } & \multicolumn{2}{|c|}{ VAT/SAT ratio } \\
\hline & Men & Women & Men & Women & Men & Women \\
\hline \multicolumn{7}{|c|}{ Age-adjusted } \\
\hline $\mathrm{BMI}$ & $0.759 * *$ & $0.725^{\star \star}$ & $0.685^{\star \star}$ & 0.618 ** & $0.317 * \star$ & 0.172 ** \\
\hline WC & $0.763^{\star *}$ & $0.635^{\star \star}$ & $0.736 * \star$ & $0.656 * \star$ & 0.380 ** & $0.265^{\star \star}$ \\
\hline WHR & $0.544^{* *}$ & $0.399 * *$ & $0.611 \star \star$ & $0.514^{\star \star}$ & $0.375^{\star \star}$ & $0.284^{\star \star}$ \\
\hline SAT & 1 & 1 & $0.619 * \star$ & $0.494^{\star \star}$ & $0.082^{\star}$ & -0.160 ** \\
\hline VAT & $0.619 * *$ & $0.494^{\star \star}$ & 1 & 1 & $0.794^{\star \star}$ & 0.726 ** \\
\hline SBP & $0.235^{\star \star}$ & $0.136 * \star$ & $0.316 * \star$ & $0.304 * *$ & $0.229 * \star$ & $0.237 * *$ \\
\hline DBP & $0.265^{\star} 8$ & $0.165^{\star \star}$ & 0.364 ** & $0.318 * \star$ & $0.278^{\star \star}$ & 0.227 ** \\
\hline FPG & $0.091^{\star}$ & $0.085^{\star}$ & $0.100 *$ & $0.126 * \star$ & 0.063 & $0.094 *$ \\
\hline $\mathrm{TG}$ & $0.121^{\star}$ & $0.092^{\star}$ & $0.259 \star \star$ & $0.276^{\star \star}$ & $0.256^{\star \star}$ & $0.262^{\star \star}$ \\
\hline HDL-C & $0.290 * \star$ & $-0.127 \star \star$ & $-0.394 * \star$ & -0.266 ** & $-0.299 \star \star$ & $-0.215^{\star \star}$ \\
\hline \multicolumn{7}{|c|}{ Age, BMI- adjusted } \\
\hline WC & $0.348 * \star$ & $0.113^{*}$ & $0.400 * \star$ & $0.335^{\star \star}$ & 0.220 ** & $0.219 * *$ \\
\hline WHR & $0.256^{\star \star}$ & 0.056 & $0.398 * \star$ & $0.297 * \star$ & $0.256^{\star \star}$ & $0.233^{* *}$ \\
\hline SAT & 1 & 1 & 0.210 ** & $0.086^{*}$ & $-0.258 * \star$ & $-0.419 * \star$ \\
\hline VAT & 0.210 ** & $0.086^{*}$ & 1 & 1 & $0.833^{\star *}$ & $0.801^{\star *}$ \\
\hline SBP & 0.049 & $-0.148 * \star$ & $0.184^{\star \star}$ & $0.143^{\star *}$ & 0.158 ** & $0.195^{\star *}$ \\
\hline DBP & $0.088^{*}$ & -0.086 * & $0.247 * \star$ & $0.173^{\star \star}$ & $0.209 * *$ & 0.186 ** \\
\hline FPG & 0.037 & -0.035 & 0.051 & 0.042 & 0.037 & 0.07 \\
\hline TG & -0.003 & $-0.113^{\star}$ & $0.205^{\star \star}$ & $0.174^{\star \star}$ & $0.218 * \star$ & $0.232^{\star *}$ \\
\hline HDL-C & $-0.085^{\star}$ & 0.059 & $-0.259 * *$ & $-0.163^{\star *}$ & $-0.220 * \star$ & $-0.183^{\star *}$ \\
\hline
\end{tabular}

Data are presented as correlation coefficients. *Pख0.05; **P $₫ 0.001$. BMI, body mass index, WC, waist circumference; WHR, waist-to-hip ratio; SAT, subcutaneous adipose tissue; VAT, visceral adipose tissue; SBP, systolic blood pressure; DBP, diastolic blood pressure; FPG, fasting plasma glucose; TG, triglyceride; HDL-C, high density lipoprotein cholesterol.

Table 3. The gender-specific associations of Z-score-transformed SAT and VAT, and VAT/SAT ratio with the metabolic abnormalities. 


\begin{tabular}{|c|c|c|c|c|c|c|c|c|c|}
\hline & \multicolumn{4}{|l|}{ Men } & \multicolumn{4}{|l|}{ Women } & \multirow{2}{*}{$\begin{array}{l}P \text { for sex } \\
\text { interaction }\end{array}$} \\
\hline & $\mathrm{MV}$ & $\mathrm{P}$ & $\mathrm{MV}+\mathrm{BMI}$ & $P$ & MV & $\mathrm{P}$ & MV +BMI & $\mathrm{P}$ & \\
\hline \multicolumn{10}{|l|}{ SAT } \\
\hline High TG & $\begin{array}{l}1.40(1.18 \\
1.65)\end{array}$ & $<0.001$ & $\begin{array}{l}1.03(0.80 \\
1.33)\end{array}$ & 0.799 & $\begin{array}{l}1.17(0.99 \\
1.38)\end{array}$ & 0.063 & $\begin{array}{l}0.90(0.70 \\
1.14)\end{array}$ & 0.365 & 0.284 \\
\hline Low HDL-C & $\begin{array}{l}1.42(1.15 \\
1.74)\end{array}$ & 0.001 & $\begin{array}{l}1.20(0.88 \\
1.62)\end{array}$ & 0.251 & $\begin{array}{l}1.16(0.97 \\
1.40)\end{array}$ & 0.107 & $\begin{array}{l}0.85(0.66 \\
1.11)\end{array}$ & 0.238 & 0.149 \\
\hline Hypertension & $\begin{array}{l}1.51(1.27 \\
1.81)\end{array}$ & $<0.001$ & $\begin{array}{l}1.08(0.83 \\
1.41)\end{array}$ & 0.546 & $\begin{array}{l}1.29(1.09 \\
1.54)\end{array}$ & 0.004 & $\begin{array}{l}0.79(0.61 \\
1.01)\end{array}$ & 0.063 & 0.225 \\
\hline $\mathrm{T} 2 \mathrm{D}$ & $\begin{array}{l}1.35(1.08 \\
1.69)\end{array}$ & 0.008 & $\begin{array}{l}1.30(0.92 \\
1.82)\end{array}$ & 0.136 & $\begin{array}{l}0.82(0.62 \\
1.08)\end{array}$ & 0.162 & $\begin{array}{l}0.48(0.33 \\
0.72)\end{array}$ & $<0.001$ & 0.008 \\
\hline \multicolumn{10}{|l|}{ VAT } \\
\hline High TG & $\begin{array}{l}2.07(1.72 \\
2.50)\end{array}$ & $<0.001$ & $\begin{array}{l}2.16(1.69, \\
2.76)\end{array}$ & $<0.001$ & $\begin{array}{l}1.53(1.29 \\
1.81)\end{array}$ & $<0.001$ & $\begin{array}{l}1.47(1.19 \\
1.83)\end{array}$ & $<0.001$ & 0.054 \\
\hline Low HDL-C & $\begin{array}{l}1.81(1.46 \\
2.25)\end{array}$ & $<0.001$ & $\begin{array}{l}1.83(1.38 \\
2.42)\end{array}$ & $<0.001$ & $\begin{array}{l}1.55(1.29 \\
1.86)\end{array}$ & $<0.001$ & $\begin{array}{l}1.47(1.17 \\
1.85)\end{array}$ & 0.001 & 0.264 \\
\hline Hypertension & $\begin{array}{l}1.96(1.62 \\
2.37)\end{array}$ & $<0.001$ & $\begin{array}{l}1.77(1.39 \\
2.26)\end{array}$ & $<0.001$ & $\begin{array}{l}1.70(1.43 \\
2.03)\end{array}$ & $<0.001$ & $\begin{array}{l}1.41(1.13 \\
1.76)\end{array}$ & 0.002 & 0.358 \\
\hline $\mathrm{T} 2 \mathrm{D}$ & $\begin{array}{l}1.20(0.96 \\
1.51)\end{array}$ & 0.114 & $\begin{array}{l}\text { 1.02(0.75, } \\
1.39)\end{array}$ & 0.91 & $\begin{array}{l}1.38(1.07 \\
1.77)\end{array}$ & 0.012 & $\begin{array}{l}1.35(0.98 \\
1.86)\end{array}$ & 0.069 & 0.393 \\
\hline \multicolumn{10}{|l|}{ VAT/SAT ratio } \\
\hline High TG & $\begin{array}{l}2.15(1.77 \\
2.60)\end{array}$ & $<0.001$ & $\begin{array}{l}2.00(1.64, \\
2.43)\end{array}$ & $<0.001$ & $\begin{array}{l}1.50(1.26 \\
1.77)\end{array}$ & $<0.001$ & $\begin{array}{l}1.45(1.22 \\
1.72)\end{array}$ & $<0.001$ & 0.013 \\
\hline Low HDL-C & $\begin{array}{l}1.66(1.35 \\
2.04)\end{array}$ & $<0.001$ & $\begin{array}{l}1.57(1.27 \\
1.94)\end{array}$ & $<0.001$ & $\begin{array}{l}\text { 1.42(1.20, } \\
1.69)\end{array}$ & $<0.001$ & $\begin{array}{l}1.37(1.15 \\
1.64)\end{array}$ & $<0.001$ & 0.275 \\
\hline Hypertension & $\begin{array}{l}1.69(1.41 \\
2.02)\end{array}$ & $<0.001$ & $\begin{array}{l}1.53(1.27 \\
1.85)\end{array}$ & $<0.001$ & $\begin{array}{l}1.54(1.29 \\
1.83)\end{array}$ & $<0.001$ & $\begin{array}{l}1.44(1.21 \\
1.73)\end{array}$ & $<0.001$ & 0.515 \\
\hline $\mathrm{T} 2 \mathrm{D}$ & $\begin{array}{l}1.03(0.82 \\
1.30)\end{array}$ & 0.785 & $\begin{array}{l}0.96(0.75 \\
1.22)\end{array}$ & 0.715 & $\begin{array}{l}1.75(1.39 \\
2.21)\end{array}$ & $<0.001$ & $\begin{array}{l}1.72(1.36, \\
2.18)\end{array}$ & $<0.001$ & 0.002 \\
\hline
\end{tabular}

Data was presented as OR $(95 \% \mathrm{Cl})$. MV adjusted for age, smoke, drink, and menstrual history (for women); SAT, subcutaneous adipose tissue; VAT, visceral adipose tissue; TG, total triglyceride; HDL-C, high density lipoprotein cholesterol; T2D, type 2 diabetes. OR, odds ratio; $\mathrm{Cl}$, confidence interval. P for sex interaction adjusted age, smoke, drink, and menstrual history (for women).

\section{Figures}


Figure 1A

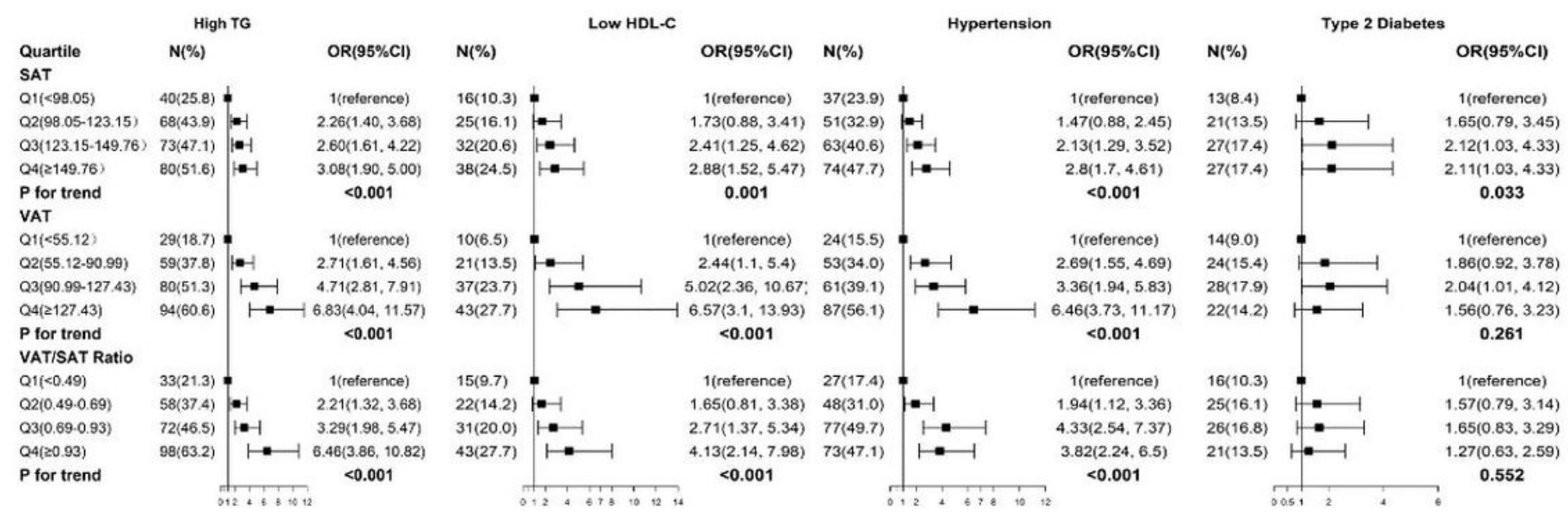

Figure 1B

\begin{tabular}{|c|c|c|c|}
\hline \multicolumn{4}{|c|}{ High TG } \\
\hline Quartile & $N(\%)$ & & OR(95\%CI) \\
\hline \multicolumn{4}{|l|}{ SAT } \\
\hline$Q 1(<139.08)$ & $38(19.9)$ & - & 1(reference) \\
\hline $\mathrm{Q} 2(139.8-178.20)$ & $45(23.6)$ & $1 *-1$ & $1.20(0.73,1.98)$ \\
\hline $\mathrm{Q} 3(178.20-221.63)$ & $54(28.0)$ & $t=-1$ & $1.45(0.90,2.36)$ \\
\hline$Q 4(2221.63)$ & $60(31.4)$ & $-1=-1$ & $1.47(0.91,2.39)$ \\
\hline P for trend & & & 0.086 \\
\hline \multicolumn{4}{|l|}{ VAT } \\
\hline Q1 $(<43.03)$ & $23(12.0)$ & - & 1(reference) \\
\hline$Q 2\{43.03-60.36)$ & $40(20.8)$ & --1 & $1.60(0.91,2.82)$ \\
\hline $\mathrm{Q} 3(60.36-79.62)$ & $60(31.3)$ & $\mapsto-1$ & $2.77(1.61,4.77)$ \\
\hline $04(279.62)$ & $74(38.7)$ & $\longmapsto$ & $-13.49(2.03,6.01)$ \\
\hline$P$ for trend & & & $<0.001$ \\
\hline \multicolumn{4}{|l|}{ VAT/SAT ratio } \\
\hline Q1 $(<0.25)$ & $25(13.1)$ & - & 1(reference) \\
\hline$Q 2(0.25-0.33)$ & $43(22.4)$ & $1-1$ & $1.77(1.02,3.07)$ \\
\hline $03(0.33-0.46)$ & $58(30.2)$ & $\mapsto-1$ & $2.46(1.45,4.18)$ \\
\hline $04(20.46)$ & $71(372)$ & $\mapsto$ & $3.34(1.98,5.64)$ \\
\hline$P$ for trend & & & $<0.001$ \\
\hline
\end{tabular}
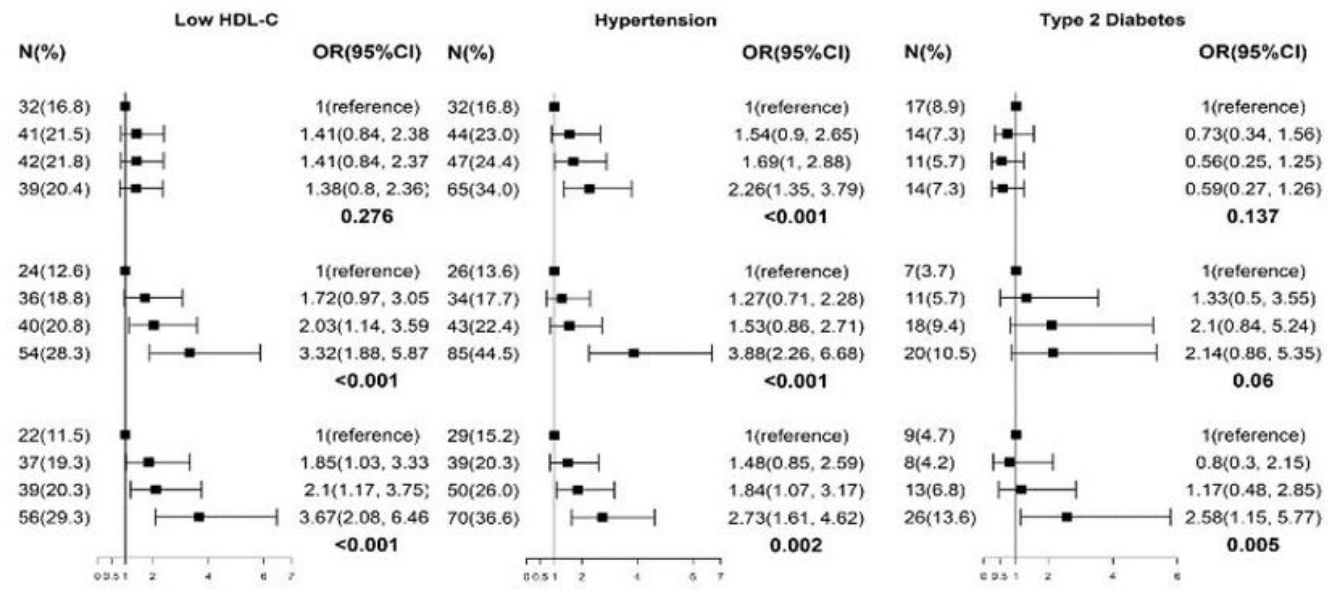

Figure 1

The associations between SAT, VAT, VAT/SAT and the risks for high TG, low HDL-C, hypertension, and Type 2 Diabetes in men(A) and women(B). N means the numbers of individuals with metabolic abnormalities. \% means the prevalence rate for metabolic abnormality. The ORs were adjusted for age, smoke, drink, menstrual history (for women). OR, odds ratio; $\mathrm{Cl}$, confidence intervals; SAT, subcutaneous adipose tissue; VAT, visceral adipose tissue; TG, total triglyceride; HDL-C, high density lipoprotein cholesterol. 


\section{Figure 2A}

\begin{tabular}{|c|c|c|c|}
\hline \multicolumn{4}{|c|}{ High TG } \\
\hline Quartile & $\mathrm{N}(\%)$ & & OR(95\%Cl) \\
\hline \multicolumn{4}{|l|}{ SAT } \\
\hline Q1(<98.05) & $40(25.8)$ & - & 1(Reference) \\
\hline Q2(98.05-123.15) & $68(43.9)$ & -1 & $1.78(1.06,2.99)$ \\
\hline $\mathrm{Q} 3(123.15-149.76)$ & $73(47.1)$ & $\bullet$ & $1.77(1.00,3.12)$ \\
\hline Q4(2149.76) & $80(51.6)$ & 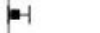 & $1.69(0.87 .3 .3)$ \\
\hline P for trend & & & 0.203 \\
\hline \multicolumn{4}{|l|}{ VAT } \\
\hline Q1(<55.12) & $29(18.7)$ & - & 1 (Reference) \\
\hline Q2(55.12-90.99) & $59(37.8)$ & $1-1$ & $2.74(1.57 .4 .76)$ \\
\hline Q3(90.99-127.43) & $80(51.3)$ & $\mapsto-1$ & $4.79(2.62 .8 .73)$ \\
\hline$Q 4(2127.43)$ & $94(60.6)$ & $\mapsto$ & $6.97(3.63,13.41)$ \\
\hline P for trend & & & $<0.001$ \\
\hline \multicolumn{4}{|l|}{ VAT/SAT Ratio } \\
\hline Q1 $(<0.49)$ & $33(21.3)$ & - & 1(Reference) \\
\hline Q2(0.49-0.69) & $58(37.4)$ & -1 & $1.79(1.05,3.05)$ \\
\hline $\mathrm{Q} 3(0.69-0.93)$ & $72(46.5)$ & $1 \cdot-1$ & $2.53(1.48,4.34)$ \\
\hline$Q 4(\geq 0.93)$ & $98(63.2)$ & $\mapsto--1$ & $5.10(2.98,8.73)$ \\
\hline$P$ for trend & & & $<0.001$ \\
\hline
\end{tabular}
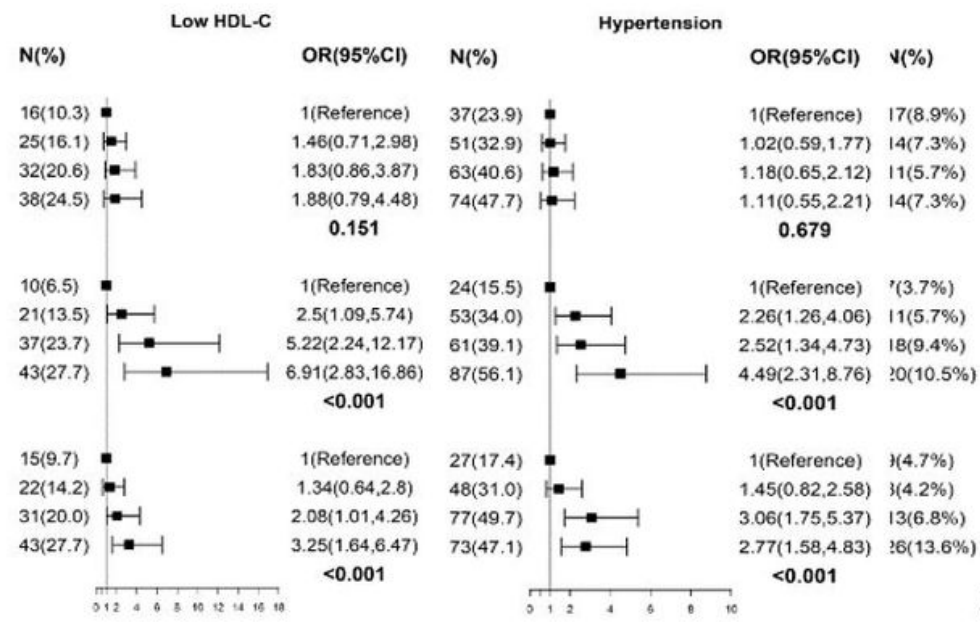

Type 2 Diabetes

OR(95\% $\mathrm{Cl})$
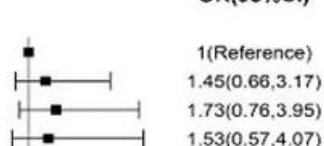

0.4

1(Reference)

$1.49(0.7,3.17)$

$1.43(0.64,3.23)$

$0.98(0.4,2.42)$

0.732

1(Reference)

$1.31(0.64 .2 .69)$

$1.32(0.64,2.74)$

$1.04(0.49,2.18)$

0.958

\section{Figure 2B}
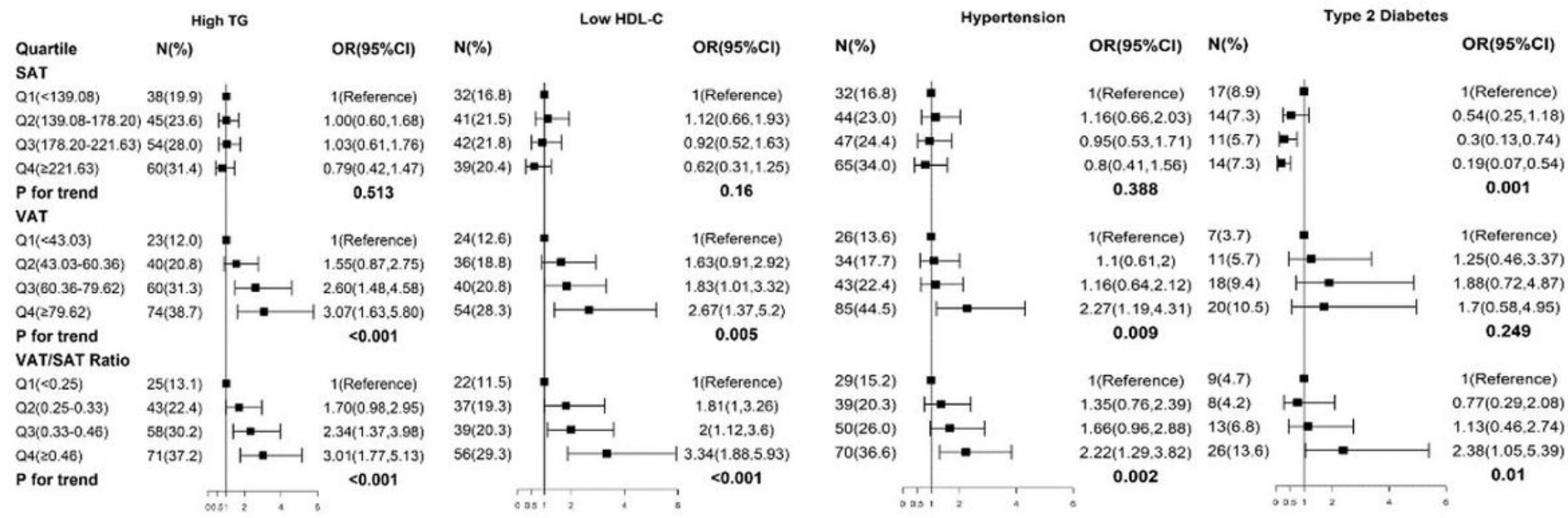

\section{Figure 2}

The associations between SAT, VAT, VAT/SAT and the risks for high TG, low HDL-C, hypertension, and Type 2 Diabetes in men $(A)$ and women(B). N means the numbers of individuals with metabolic abnormalities. \% means the prevalence rate for metabolic abnormality. The ORs were adjusted for age, BMI, smoke, drink, menstrual history (for women). OR, odds ratio; $\mathrm{Cl}$, confidence intervals; SAT, subcutaneous adipose tissue; VAT, visceral adipose tissue; TG, total triglyceride; HDL-C, high density lipoprotein cholesterol. 